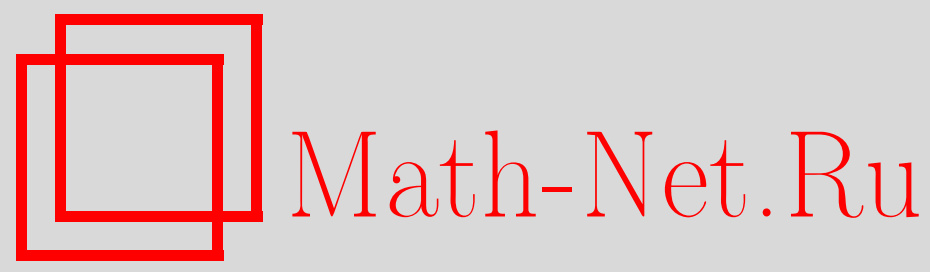

А. М. Адамович, Строение модулей когомологий линейных расслоений на $G / B$ и слабый порядок на группе Вейля, $M a$ тем. заметки, 1997, том 62, выпуск 2, 163-168

DOI: https://doi.org/10.4213/mzm1601

Использование Общероссийского математического портала Math-Net.Ru подразумевает, что вы прочитали и согласны с пользовательским соглашением http://www.mathnet.ru/rus/agreement

Параметры загрузки:

IP: 35.174 .16 .151

26 апреля 2023 г., 02:34:33

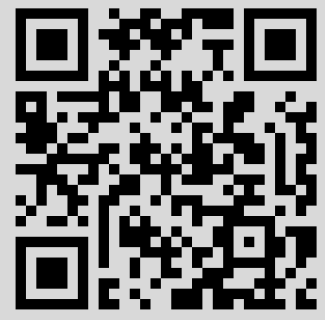




\section{СТРОЕНИЕ МОДУЛЕЙ КОГОМОЛОГИЙ ЛИНЕЙНЫХ РАССЛОЕНИЙ НА $G / B$ И СЛАБЫЙ ПОРЯДОК НА ГРУППЕ ВЕЙЛЯ}

\section{А. М. Адамович}

Рассматриваются $G$-модули когомологий линейных расслоений на многообразии флагов $G / B$ над алгебраически замкнутым полем простой характеристики. Изучаются строение подмодулей этих модулей и связьвающие их гомоморфизмы.

Библиография: 3 названия.

Пусть $G$ - связная односвязная полупростая алгебраическая групша над алгебраически замкнутым полем $k$ характеристики $p>0 ; T$ - ее максимальньй тор; $R$-система корней, соответствуюшая паре $(G, T) ; \Delta$ - множество простых корней; $R_{+}\left(R_{-}\right)$- множества положительных (соответственно отрицательных) корней; $B \supset T$ - борелевская подгруппа, соответствуюшая $R_{-} ; X-$ группа характеров $T ; X^{+}=\left\{\lambda \in X \mid 0 \leqslant\left\langle\alpha^{\vee}, \lambda\right\rangle\right.$ для $\alpha \in \Delta\}$ - множество доминантных весов; $X_{n}=\left\{\lambda \in X \mid 0 \leqslant\left\langle\alpha^{\vee}, \lambda\right\rangle<p^{n}\right.$ для $\alpha \in \Delta\}$ - множество $p^{n}$-ограниченньх весов; $L(\lambda)$ - простой $G$-модуль со старшим весом $\lambda \in X^{+} ; W$ - группа Вейля; $l(w)$ - длина элемента $w \in W$ относительно множества простых отражений $S=\left\{s_{\alpha} \mid \alpha \in \Delta\right\} ; w_{0}$ - максимальньй элемент $W$; $w \cdot \lambda=w(\lambda+\rho)-\rho$, где $\rho$ - полусумма положительных корней.

Будем изучать строение $G$-модулей $H^{i}(y \cdot \lambda)=H^{i}(G / B, \mathscr{L}(y \cdot \lambda))$, где $\mathscr{L}(y \cdot \lambda)$ - линейное расслоение на $G / B$, индуцированное характером $y$. $\lambda$ групшы $B, y \in W, \lambda \in X^{+}$. В общем случае $H^{i}(y \cdot \lambda)=0$ при $i \neq l(y)$, а модули $H^{l(y)}(y . \lambda)$ неразложимы и характер каждого из них равен характеру Вейля $\chi(\lambda)$. При этом $H^{l\left(w_{0}\right)}\left(w_{0} \cdot \lambda\right)$ изоморфен модулю Вейля $V(\lambda)$, т.е. универсальному $G$-модулю, порожденному вектором старшего веса $\lambda$. Так как характеры одинаковы, то все $H^{l(y)}(y . \lambda)$ имеют одно и то же множество композиционных факторов. Однако, эти модули различаются строением подмодулей.

В [1] Андерсен доказал, что модули $H^{l(y)}(y . \lambda)$ имеют однократные "несушие" композиционные факторы $L\left(\lambda^{w}\right)$ (множество несущих факторов параметризуется элементами группы Вейля). Эти факторы интересны тем, что $L\left(\lambda^{w_{0} y}\right)$ является первым, а $L\left(\lambda^{y}\right)-$ последним фактором в любом убывающем композиционном ряду модуля $H^{l(y)}(y \cdot \lambda)$ (это означает, что $\operatorname{Soc} H^{l(y)}(y . \lambda) \simeq L\left(\lambda^{y}\right)$, a $\left.H^{l(y)}(y \cdot \lambda) / \operatorname{Rad} H^{l(y)}(y \cdot \lambda) \simeq L\left(\lambda^{w_{0} y}\right)\right)$.

Возникает вопрос о расположении остальных несущих факторов в композиционных рядах $H^{l(y)}(y \cdot \lambda)$. Каждому $H^{l(y)}(y \cdot \lambda)$ мы можем поставить в соответствие частичное упорядочение на множестве $\left\{L\left(\lambda^{w}\right)\right\}$ несущих факторов, полагая $L\left(\lambda^{w_{2}}\right)>_{y} L\left(\lambda^{w_{1}}\right)$, если в любом убывающем композиционном ряду $H^{l(y)}(y \cdot \lambda)$ фактор $L\left(\lambda^{w_{2}}\right)$ встречается раньше, чем $L\left(\lambda^{w_{1}}\right)$.

Работа вьполненапри финансовой поддержке Международного научного фонда, грант M7N000. 
Основной результат (теорема 3 ) настоящей работы состоит в том, что в общем случае $L\left(\lambda^{w_{2}}\right)>_{y} L\left(\lambda^{w_{1}}\right)$ эквивалентно $w_{2} y^{-1}>w_{1} y^{-1}$ (в смысле слабого порядка на группе Вейля). Используемые методы также позволяют в более общей ситуации доказать утверждение Андерсена (см. $[1,2.4])$ о гомоморфизмах между $H^{l(y)}(y . \lambda)$ для различных $y \in W$.

Напомним как определяется отношение слабого порядка (см., например, [2]) на $W$ : $w \leqslant \widetilde{w}$, если $l\left(\widetilde{w} w^{-1}\right)=l(\widetilde{w})-l(w)$, т.е. если $\widetilde{w}$ можно представить в виде произведения $\widetilde{w}=s_{1} \cdots s_{r} w$, где $s_{i} \in S, r=l(\widetilde{w})-l(w)$.

Введем необходимые обозначения.

Пусть $\lambda \in X^{+}$и $n$ - такое, что $\lambda \in X_{n+1} \backslash X_{n}$. Существует единственное разложение $\lambda=\lambda^{0}+p^{n} \lambda^{1}$, где $\lambda^{0} \in X_{n}$ и $\lambda^{1} \in X^{+}$. Следуя Андерсену [1], будем называть вес $\lambda \in X^{+}$общим, если

$$
6(h-1) \leqslant\left\langle\alpha^{\vee}, \lambda^{1}\right\rangle \leqslant p-6(h-1) \quad \text { для всех } \alpha \in R_{+}
$$

(число Кокстера $h=\left\langle\alpha_{0}^{\vee}, \rho\right\rangle+1$, если $R$ неразложима, а $\alpha_{0}$-максимальньй короткий корень; в общем случае $h$ - максимальноеиз чисел Кокстера неразложимых компонент $R$ ).

Всюду, далее, $\lambda \in X^{+}{ }_{-}$общий вес.

Каждьй элемент $\mu \in X$ имеет единственное разложение $\mu=\mu^{0}+p^{n} \mu^{1}$, где $\mu^{0} \in X_{n}$ и $\mu^{1} \in X$. Для общего веса $\lambda \in X_{n+1} \backslash X_{n}$ и $w \in W$ положим

$$
\lambda^{w}=(w \cdot \lambda)^{0}+p^{n} w^{-1} \cdot\left((w \cdot \lambda)^{1}\right)
$$

Через Soc $M$ будем обозначать максимальньй полупростой подмодуль модуля $M$, a через $\operatorname{Rad} M$ - минимальный подмодуль модуля $M$, фактор по которому полупрост.

Нам понадобятся доказанные в [1] свойства модулей $H^{l(y)}(y . \lambda)$ для $y \in W$ :

1) $\left[H^{l(y)}(y . \lambda): L\left(\lambda^{w}\right)\right]=1$ для $w \in W$;

2) $\operatorname{Soc} H^{l(y)}(y \cdot \lambda) \simeq L\left(\lambda^{y}\right)$;

3) $H^{l(y)}(y \cdot \lambda) / \operatorname{Rad} H^{l(y)}(y \cdot \lambda) \simeq L\left(\lambda^{w_{0} y}\right)$.

Так как $\left[H^{l(y)}(y \cdot \lambda): L\left(\lambda^{w}\right)\right]=1$, существует единственный минимальньй подмодуль модуля $H^{l(y)}(y \cdot \lambda)$, имеюший $L\left(\lambda^{w}\right)$ композиционным фактором. Обозначим его через $H^{l(y)}(y . \lambda)^{w}$.

Отметим, что вследствие свойств 2) и 3) модулей когомологий $\operatorname{Soc} H^{l(y)}(y . \lambda)=$ $H^{l(y)}(y \cdot \lambda)^{y}$ и $H^{l(y)}(y \cdot \lambda)=H^{l(y)}(y \cdot \lambda)^{w_{0} y}$.

Очевидно, что $H^{l(y)}(y \cdot \lambda)^{w_{1}} \subset H^{l(y)}(y \cdot \lambda)^{w_{2}}$ тогда и только тогда, когда в любом убывающем композиционном ряду модуля $H^{l(y)}(y . \lambda)$ фактор $L\left(\lambda^{w_{2}}\right)$ встречается раньше, чем $L\left(\lambda^{w_{1}}\right)$.

Основным инструментом изучения строения модулей когомологий $H^{l(y)}(y . \lambda)$ служат морфизмы между ними. Андерсен в работе [3] определил для $y \in W, s \in S$ ненулевой морфизм

$$
T_{s}: H^{l(y)}(y \cdot \lambda) \rightarrow H^{l(s y)}(s y \cdot \lambda) .
$$

Если $l(w)>1$ и $w=s_{1} \cdots s_{r}-$ приведенное разложение, то морфизм

$$
T_{w}: H^{l(y)}(y \cdot \lambda) \rightarrow H^{l(w y)}(w y \cdot \lambda)
$$

определяется как композиция $T_{s_{1}} \cdots T_{s_{r}}$ (результат не зависит от выбора приведенного разложения), $T_{e}$ - тождественньй автоморфизм. Заметим, что если $w \leqslant \widetilde{w}$, то $T_{\widetilde{w}}=T_{\widetilde{w} w^{-1}} T_{w}$.

Из $[3,4.4]$ непосредственно следует 
ПрЕДЛОЖЕНИЕ (Андерсен). Морфизм $T_{y^{-1}}: H^{l(y)}(y . \lambda) \rightarrow H^{0}(\lambda)$ не является нулевы.м.

Из определения подмодулей вида $H^{l(y)}(y \cdot \lambda)^{w}$ легко следуют два утверждения, которые удобно сформулировать в виде лемм.

Лемма 1. Пусть $\varphi \in \operatorname{Hom}\left(H^{l(y)}(y \cdot \lambda), H^{l(w)}(w \cdot \lambda)\right), z \in W$. Тогдa

$$
\text { или } \quad \varphi H^{l(y)}(y \cdot \lambda)^{z}=H^{l(w)}(w \cdot \lambda)^{z}, \quad \text { или } \quad H^{l(y)}(y \cdot \lambda)^{z} \subseteq \operatorname{Ker} \varphi .
$$

В частности, если $\varphi \neq 0$, mо $\varphi H^{l(y)}(y \cdot \lambda)=H^{l(w)}(w \cdot \lambda)^{w_{0} y}$.

Лемма 2. Пусть $\varphi \in \operatorname{Hom}\left(H^{l(y)}(y \cdot \lambda), H^{l(w)}(w \cdot \lambda)\right), z \in W$. Тогда если

$$
H^{l(w)}(w \cdot \lambda)^{z} \subseteq \operatorname{Im} \varphi
$$

mo

$$
\varphi H^{l(y)}(y \cdot \lambda)^{z}=H^{l(w)}(w \cdot \lambda)^{z} .
$$

В частности, если $\varphi \neq 0$, mo $\varphi H^{l(y)}(y \cdot \lambda)^{w}=\operatorname{Soc} H^{l(w)}(w \cdot \lambda)$.

Teорема 1. Справедливо равенство $\operatorname{Hom}\left(H^{l(y)}(y \cdot \lambda), H^{l(w)}(w \cdot \lambda)\right)=k$. Mopфизм $T_{w y^{-1}}: H^{l(y)}(y \cdot \lambda) \rightarrow H^{l(w)}(w \cdot \lambda)$ является ненулевым.

ЗАмечАниЕ. В [1] эта теорема доказана для $n=1$.

ДокаЗАТЕЛЬСТво ТЕОРемЫ 1 . Так как $H^{l(w)}(w \cdot \lambda)$ имеет простой цоколь, изоморфный $L\left(\lambda^{w}\right)$, то

$$
\operatorname{dim} \operatorname{Hom}\left(H^{l(y)}(y \cdot \lambda), H^{l(w)}(w \cdot \lambda)\right) \leqslant\left[H^{l(y)}(y \cdot \lambda): L\left(\lambda^{w}\right)\right]=1 .
$$

Поэтому достаточно проверить, что $T_{w y^{-1}} \neq 0$.

Так как $w y^{-1} \leqslant w_{0}$, то диаграмма

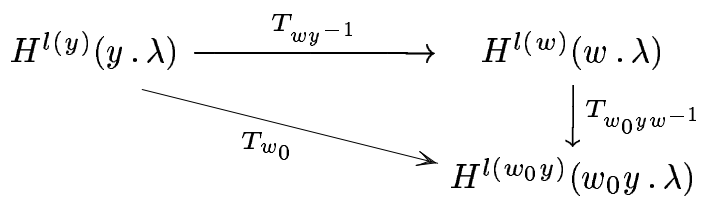

коммутативна. Видно, что если $T_{w_{0}} \neq 0$, то и $T_{w y^{-1}} \neq 0$. Осталось доказать нетривиальность морфизма

$$
T_{w_{0}}: H^{l(y)}(y \cdot \lambda) \rightarrow H^{l\left(w_{0} y\right)}\left(w_{0} y \cdot \lambda\right) .
$$

Для этого нам понадобятся две леммы.

ЛЕмма 3. Выполнено $w_{1} \leqslant w_{2} \Longrightarrow H^{0}(\lambda)^{w_{1}} \subseteq H^{0}(\lambda)^{w_{2}}$. 
ДокАЗАТЕЛЬСтво. Пусть $w_{1} \leqslant w_{2}$. По определению это означает, что $l\left(w_{2} w_{1}^{-1}\right)=$ $l\left(w_{2}\right)-l\left(w_{1}\right)$. Используя равенства $l\left(w_{0} z\right)=l\left(z w_{0}\right)=l\left(w_{0}\right)-l(z)$ и $l(z)=l\left(z^{-1}\right)$, нетрудно проверить, что $l\left(w_{2}^{-1} w_{0}\right)=l\left(w_{1}^{-1} w_{0}\right)-l\left(w_{0} w_{2} w_{1}^{-1} w_{0}\right)$, т.е. $w_{0} w_{2} w_{1}^{-1} w_{0} \leqslant w_{1}^{-1} w_{0}$. Поэтому мы имеем коммутативную диаграмму

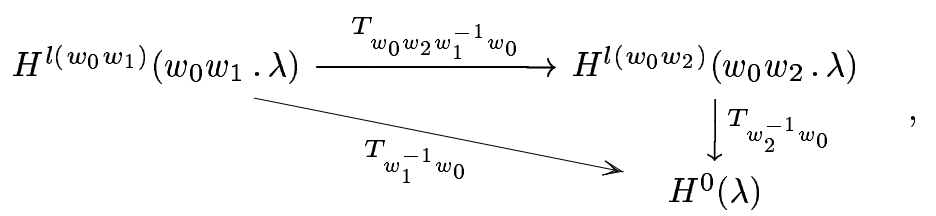

причем $T_{w_{i}^{-1} w_{0}} \neq 0$ (см. предложение) для $i=1,2$. Следовательно, по лемме 1 вьполнено $T_{w_{i}^{-1} w_{0}} H^{l\left(w_{0} w_{i}\right)}\left(w_{0} w_{i} \cdot \lambda\right)=H^{0}(\lambda)^{w_{i}}$. Но

$$
T_{w_{1}^{-1} w_{0}} H^{l\left(w_{0} w_{1}\right)}\left(w_{0} w_{1} \cdot \lambda\right) \subseteq T_{w_{2}^{-1} w_{0}} H^{l\left(w_{0} w_{2}\right)}\left(w_{0} w_{2} \cdot \lambda\right)
$$

ввиду коммутативности диаграммы.

Лемма 4. Морфизм $T_{w}: H^{0}(\lambda) \rightarrow H^{l(w)}(w . \lambda)$ не является нулевым.

ДоКАЗАТЕЛЬСтво. Воспользуемся индукцией по $l(w)$. Для $l(w)=1$ утверждение леммы вьполнено, так как $T_{w}=T_{s}$ - ненулевой. Пусть $w=s w_{1}, l(w)=l\left(w_{1}\right)+1, T_{w_{1}}-$ ненулевой, диаграмма

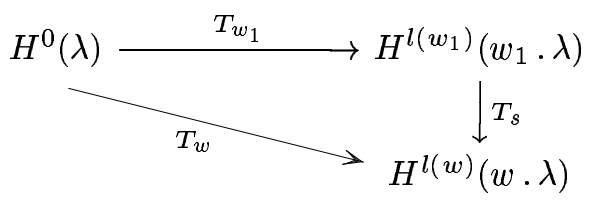

коммутативна.

Так как $T_{w_{1}} \neq 0$, то вследствие леммы $2 T_{w_{1}} H^{0}(\lambda)^{w_{1}}=\operatorname{Soc} H^{l\left(w_{1}\right)}\left(w_{1} \cdot \lambda\right)$. Далее, Soc $H^{l\left(w_{1}\right)}\left(w_{1} \cdot \lambda\right) \simeq L\left(\lambda^{w_{1}}\right)$, поэтому $\left[T_{w_{1}} H^{0}(\lambda): L\left(\lambda^{w_{1}}\right)\right]=1$. По лемме 3 в любом убывающем композиционном ряду $G$-модуля $H^{0}(\lambda)$ фактор $L\left(\lambda^{w}\right)$ предшествует $L\left(\lambda^{w_{1}}\right)$ (или они совпадают в случае $\left.\lambda^{w}=\lambda^{w_{1}}\right)$. Следовательно, в любом композиционном ряду модуля $T_{w_{1}} H^{0}(\lambda)$ фактор $L\left(\lambda^{w}\right)$ также встречается, и $H^{l\left(w_{1}\right)}\left(w_{1} \cdot \lambda\right)^{w} \subseteq T_{w_{1}} H^{0}(\lambda)$.

Далее, ввиду леммы $2 \operatorname{Soc} H^{l(w)}(w \cdot \lambda)=T_{s} H^{l\left(w_{1}\right)}\left(w_{1} \cdot \lambda\right)^{w}$, поскольку $T_{s} \neq 0$. Итак, мы получаем $\operatorname{Soc} H^{l(w)}(w \cdot \lambda) \subseteq T_{s} T_{w_{1}} H^{0}(\lambda)$ и $T_{w}=T_{s} T_{w_{1}} \neq 0$.

Продолжим доказательство теоремы. Мы имеем коммутативную диаграмму

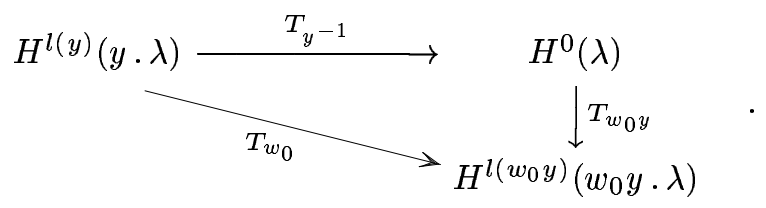

Докажем, что $T_{w_{0}} \neq 0$.

В самом деле, $T_{y^{-1}} \neq 0$ (см. предложение). Значит, $T_{y^{-1}} H^{l(y)}(y . \lambda)=H^{0}(\lambda) w_{0} y . \mathrm{C}$ другой стороны, Soc $H^{l\left(w_{0} y\right)}\left(w_{0} y \cdot \lambda\right)=T_{w_{0} y} H^{0}(\lambda) w_{0} y$, так как $T_{w_{0} y} \neq 0$ по лемме 4. Таким образом, $T_{w_{0} y} T_{y^{-1}} H^{l(y)}(y \cdot \lambda)=\operatorname{Soc} H^{l\left(w_{0} y\right)}\left(w_{0} y \cdot \lambda\right)$ и $T_{w_{0}} \neq 0$. 
TEOPEMA 2. Eсли $w_{1} y^{-1} \leqslant w_{2} y^{-1}, m o H^{l(y)}(y \cdot \lambda)^{w_{1}} \subseteq H^{l(y)}(y \cdot \lambda)^{w_{2}}$.

ДокАЗАТЕЛЬСТво аналогично доказательству леммы 3: морфизмы

$$
T_{y w_{i}^{-1} w_{0}}: H^{l\left(w_{0} w_{i}\right)}\left(w_{0} w_{i} \cdot \lambda\right) \rightarrow H^{l(y)}(y \cdot \lambda), \quad i=1,2,
$$

ненулевые по теореме 1, поэтому вследствие леммы 1

$$
H^{l(y)}(y \cdot \lambda)^{w_{i}}=T_{y w_{i}^{-1} w_{0}} H^{l\left(w_{0} w_{i}\right)}\left(w_{0} w_{i} \cdot \lambda\right),
$$

a

$$
T_{y w_{1}^{-1} w_{0}} H^{l\left(w_{0} w_{1}\right)}\left(w_{0} w_{1} \cdot \lambda\right)=T_{y w_{2}^{-1} w_{0}} T_{w_{0} w_{2} w_{1}^{-1} w_{0}} H^{l\left(w_{0} w_{1}\right)}\left(w_{0} w_{1} \cdot \lambda\right)
$$

и следовательно,

$$
T_{y w_{1}^{-1} w_{0}} H^{l\left(w_{0} w_{1}\right)}\left(w_{0} w_{1} \cdot \lambda\right) \subseteq T_{y w_{2}^{-1} w_{0}} H^{l\left(w_{0} w_{2}\right)}\left(w_{0} w_{2} \cdot \lambda\right) .
$$

Теорема 3. Пусть все $\lambda^{w}$ различны. Тогда

$$
w_{1} y^{-1} \leqslant w_{2} y^{-1} \Longleftrightarrow H^{l(y)}(y \cdot \lambda)^{w_{1}} \subseteq H^{l(y)}(y \cdot \lambda)^{w_{2}} .
$$

ДокАЗАТЕльство. Осталось доказать ‘ $\Longleftarrow$ '. В предположениях теоремы докажем сначала две леммы.

Рассмотрим морфизмы

$$
T_{s}: H^{l(w)}(w \cdot \lambda) \rightarrow H^{l(s w)}(s w \cdot \lambda) \quad \text { и } \quad T_{s}: H^{l(s w)}(s w \cdot \lambda) \rightarrow H^{l(w)}(w \cdot \lambda) .
$$

Лемма 5. Композиция $T_{s} T_{s}=0$.

ДокаЗАТЕльство. По теореме $1 T_{s} T_{s} \in \operatorname{Hom}\left(H^{l(w)}(w \cdot \lambda), H^{l(w)}(w \cdot \lambda)\right)$ скалярен. Поэтому или $T_{s} T_{s}=0$, или $T_{s}-$ изоморфизм. Последнее невозможно, так как в этом случае

$$
L\left(\lambda^{w}\right) \simeq \operatorname{Soc} H^{l(w)}(w \cdot \lambda) \simeq \operatorname{Soc} H^{l(s w)}(s w \cdot \lambda) \simeq L\left(\lambda^{s w}\right),
$$

т.e. $\lambda^{w}=\lambda^{s w}$.

Лемма 6. Пусть $s \in S, w, y \in W$. Тогдa

$$
H^{l(s y)}(s y \cdot \lambda)^{w}=T_{s} H^{l(y)}(y \cdot \lambda)^{w} \Longleftrightarrow l\left(w y^{-1} s\right)=l\left(w y^{-1}\right)-1 .
$$

ДОКАЗАТЕЛЬСТВо. Возможны два случая.

1) $l\left(w y^{-1} s\right)=l\left(w y^{-1}\right)-1$.

Тогда $T_{w y^{-1}}=T_{w y^{-1} s} T_{s}$. Вследствие теоремы $1 T_{w y^{-1}} H^{l(y)}(y \cdot \lambda) \neq 0$. Поэтому (см. лемму 2 )

$$
T_{w y^{-1}} H^{l(y)}(y \cdot \lambda)^{w}=\operatorname{Soc} H^{l(w)}(w \cdot \lambda) .
$$

Таким образом, $H^{l(y)}(y \cdot \lambda)^{w} \nsubseteq \operatorname{Ker} T_{w y^{-1}}=\operatorname{Ker} T_{w y^{-1} s} T_{s}$ и $H^{l(y)}(y \cdot \lambda)^{w} \nsubseteq \operatorname{Ker} T_{s}$. Следовательно, по лемме $1 T_{s} H^{l(y)}(y \cdot \lambda)^{w}=H^{l(s y)}(s y \cdot \lambda)^{w}$.

2) $l\left(w y^{-1} s\right)=l\left(w y^{-1}\right)+1$. 
Тогда (так как $\left.y^{-1} s=(s y)^{-1}\right)$ по доказанному выше $H^{l(y)}(y \cdot \lambda)^{w} \subseteq T_{s} H^{l(s y)}(s y \cdot \lambda)$. В силу леммы $5 H^{l(y)}(y . \lambda)^{w} \subseteq \operatorname{Ker} T_{s}$. Следовательно, $\left[T_{s} H^{l(y)}(y \cdot \lambda): L\left(\lambda^{w}\right)\right]=0$ и $H^{l(s y)}(s y \cdot \lambda)^{w} \nsubseteq T_{s} H^{l(y)}(y \cdot \lambda)$.

Теперь докажем с помощью индукции по $l=l\left(w_{1} y^{-1}\right)$, что

$$
H^{l(y)}(y \cdot \lambda)^{w_{1}} \subseteq H^{l(y)}(y \cdot \lambda)^{w_{2}} \Longrightarrow w_{1} y^{-1} \leqslant w_{2} y^{-1}
$$

т.е. $l\left(w_{2} w_{1}^{-1}\right)=l\left(w_{2} y^{-1}\right)-l\left(w_{1} y^{-1}\right)$.

Для $l=0$ это утверждение, очевидно, верно. Предположим, что оно верно для $l=r-1$.

Пусть $l\left(w_{1} y^{-1}\right)=r, w_{1} y^{-1}=s_{1} \cdots s_{r}-$ приведенное разложение. Тогда диаграмма

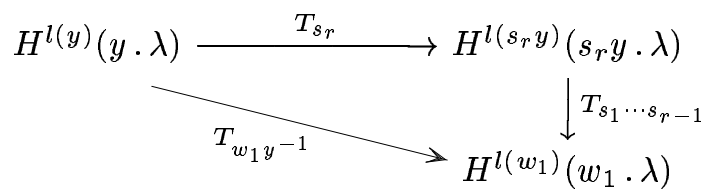

коммутативна, $T_{w_{1} y^{-1}}$ - ненулевой. Поэтому $T_{w_{1} y^{-1}} H^{l(y)}(y \cdot \lambda)^{w_{1}}=\operatorname{Soc} H^{l\left(w_{1}\right)}\left(w_{1} \cdot \lambda\right)$. Следовательно, $H^{l(y)}(y . \lambda)^{w_{1}} \nsubseteq \operatorname{Ker} T_{w_{1} y^{-1}}$, а так как $H^{l(y)}(y \cdot \lambda)^{w_{1}} \subseteq H^{l(y)}(y \cdot \lambda)^{w_{2}}$, то $H^{l(y)}(y \cdot \lambda)^{w_{2}} \nsubseteq \operatorname{Ker} T_{w_{1} y^{-1}}$ и $H^{l(y)}(y \cdot \lambda)^{w_{2}} \nsubseteq \operatorname{Ker} T_{s_{r}}$. Тогда по лемме 1

$$
T_{s_{r}} H^{l(y)}(y \cdot \lambda)^{w_{2}}=H^{l\left(s_{r} y\right)}\left(s_{r} y \cdot \lambda\right)^{w_{2}},
$$

а по лемме $6 l\left(w_{2} y^{-1} s_{r}\right)=l\left(w_{2} y^{-1}\right)-1$.

Далее, $l\left(w_{1}\left(s_{r} y\right)^{-1}\right)=l\left(w_{1} y^{-1} s_{r}\right)=r-1$, a

$$
H^{l\left(s_{r} y\right)}\left(s_{r} y \cdot \lambda\right)^{w_{1}}=T_{s_{r}} H^{l(y)}(y \cdot \lambda)^{w_{1}} \subseteq T_{s_{r}} H^{l(y)}(y \cdot \lambda)^{w_{2}}=H^{l\left(s_{r} y\right)}\left(s_{r} y \cdot \lambda\right)^{w_{2}}
$$

(первое равенство следует из леммы 6).

Отсюда по предположению индукции $l\left(w_{2} w_{1}^{-1}\right)=l\left(w_{2} y^{-1} s_{r}\right)-l\left(w_{1} y^{-1} s_{r}\right)$. Таким образом,

$$
l\left(w_{2} w_{1}^{-1}\right)=l\left(w_{2} y^{-1}\right)-1-l\left(s_{1} \cdots s_{r-1}\right)=l\left(w_{2} y^{-1}\right)-r=l\left(w_{2} y^{-1}\right)-l\left(w_{1} y^{-1}\right)
$$

т.е. $w_{1} y^{-1} \leqslant w_{2} y^{-1}$ и при $l=r$.

\section{СПИСОК ЦИТИРОВАННОЙ ЛИТЕРАТУРЫ}

[1] Andersen H. H. On the generic structure of cohomology modules for semi-simple algebraic groups // Trans. Amer. Math. Soc. 1986. V. 295. P. 397-415.

[2] Björner A. Orderings of Coxeter groups // Contemp. Math. 1984. V. 34. P. 175-195.

[3] Andersen H. H. Filtrations of cohomology modules for Chevalley groups // Ann. Sci. École Norm. Sup. 1983. V. 16. №4. P. 495-528. 